\title{
ESTRATEGIA DE DISTRIBUCIÓN DEL PISCO PERUANO EN EL MERCADO DE ESTADOS UNIDOS: EL CASO DE TEXAS*
}

\author{
Natali Lama \\ natalilama@gmail.com \\ Anahí Martínez \\ acmr72@hotmail.com \\ Richard Rosado \\ rrosadoli@hotmail.com
}

\begin{abstract}
Resumen
En este trabajo se evalúan los canales de distribución del pisco en el mercado de licores de Estados Unidos para diseñar una estrategia de ingreso y comercialización de este producto enfocada específicamente en el estado de Texas. Para lograr este objetivo, se procede a estudiar el tamaño del mercado de bebidas espirituosas, las características y las tendencias del consumo, el tamaño y las características de la oferta exportable, las estrategias de comercialización emprendidas por los actuales exportadores, así como las consideraciones legales y tributarias que regulan la comercialización de licores en ese país. Finalmente, se analiza la cadena de distribución, desde su estructura de costos hasta los beneficios y márgenes por participante. La investigación comprendió data estadística, investigación de campo en mercados de destino, entrevistas a expertos estadounidenses, prueba de producto, estudio de patrones de comportamiento de clusters de exportadores, entre otros aspectos.
\end{abstract}

Palabras clave: distribución, canales, estrategia, exportación, pisco, Estados Unidos.

\begin{abstract}
In this piece, the distribution channels for pisco-Peruvian first press grape brandy-in the United States liquor market are evaluated in order to design entry and commercialization strategies for this product, focusing specifically on the state of Texas. To meet this objective, studies are conducted for the size of the spirited drinks market, the characteristics and trends of consumption, the size and characteristics of the export supply, commercialization strategies being used by current exporters, as well as legal and tax considerations that regulate the commercialization of liquor in the US. Finally, the distribution chain is analyzed, from the costs structure to the benefits and margins per participant. The investigation covered statistical data, field investigations for destination markets, interviews with American experts, product testing and pattern studies on the behavior of exporters' clusters, among other aspects.
\end{abstract}

Key words: distribution, channels, strategy, exportation, pisco, United States.

* Basado en la tesis del mismo título presentada por los autores a la Universidad ESAN en el 2006 para optar de grado de Magíster en Administración. 


\section{Introducción}

La magia de los mercados internacionales cautiva cada vez a mayor número de agentes económicos. Sin embargo, en muchas ocasiones el tema es esquivo por el desconocimiento de los posibles mercados. Detectada esta problemática, se decidió realizar el ejercicio de cartografiar un mercado extranjero para un producto peruano en particular, con énfasis en los canales de distribución.

El producto escogido fue el pisco; y el país, Estados Unidos. Se eligió este mercado porque se pudo obtener la información necesaria durante un viaje de intercambio académico a Texas. Por tanto, el pisco y su distribución en Estados Unidos es el eje de este trabajo.

Cabe señalar que esta investigación sigue la línea de las tesis presentadas anteriormente a la Universidad ESAN cuyos temas han girado en torno al comercio internacional, como el plan de negocios para la exportación de joyería de diseño al mercado estadounidense y la propuesta para desarrollar la cadena del pisco peruano con miras al mercado global.

El pisco peruano es una bebida alcohólica de elevada calidad. A pesar de ello, hasta hace poco su participación en el mercado, externo e interno, no fue significativa. Recién en los últimos años ha aprovechado las oportunidades favorables, como lo demuestra el comportamiento de sus exportaciones a Estados Unidos.

El conocimiento y el aprovechamiento de la cadena de distribución constituyen los factores críticos para participar en este mercado, razón por la cual se procedió a analizar aspectos legales, agentes partici- pantes, márgenes, carga tributaria, entre otros factores.

Posteriormente, se realizó una evaluación financiera de las posibilidades de utilizar diferentes diseños de canales de distribución dentro de la cadena. Se evaluó la factibilidad de acortar los intermediarios mediante la inversión directa en un importador-distribuidor en territorio estadounidense. Esta posibilidad genera un mejor resultado económico para el empresario peruano.

\section{El producto}

\subsection{Definición del pisco}

Según la norma técnica peruana del Instituto Nacional de Defensa de la Competencia y de la Protección de la Propiedad Intelectual (Indecopi):

El pisco es el aguardiente obtenido exclusivamente por destilación de mostos frescos de «Uvas Pisqueras», recientemente fermentados, utilizando métodos que mantengan el principio tradicional de calidad establecido en las zonas de producción reconocidas. ... Es una bebida alcohólica de color transparente o ligeramente ambarino, con un contenido de alcohol de $42^{\circ}$ en promedio (Indecopi, 2006).

En el ámbito internacional, si bien el pisco es consumido por las colonias peruanas como un producto étnico, su calidad es tal que permite su comercialización fuera de este circuito. Según Johnny Schuler, actual presidente de la Cofradía de Catadores del Perú: «... el pisco tiene una excelente calidad que ha sido aceptada por reconocidos catadores en el nivel mundial ${ }^{1}$, lo que

1. Entrevista realizada en Lima, el 19 de enero de 2006. 
lo ha hecho acreedor a diversos premios internacionales. Respalda esta aseveración el experto en licores Diego Loret de Mola ${ }^{2}$, quien indica: «... expertos en Estados Unidos ya califican el pisco peruano como un licor de excepcional grado internacional, y afirman que tiene gran potencial para establecerse como nueva categoría de licores en dicho país».

La revista Wine Enthusiast, considerada una de las principales guías de bebidas alcohólicas en Estados Unidos, coloca una marca de pisco peruano entre las cien mejores bebidas alcohólicas y la define como una bebida «altamente recomendable» (Pacult, 2005).

\subsection{Situación actual del sector del pisco en el Perú}

Entre algunos datos relevantes sobre la producción de pisco, puede mencionarse que en el año 2004 se presentó un incremento de la producción de $23 \%$ con respecto al 2003, y que en el 2005 el aumento fue de $36 \%$ con relación al año anterior. Las cifras oficiales indican que en el 2004 se produjeron 600 mil litros; y en el 2005, aproximadamente $814 \mathrm{mil} \mathrm{litros}^{3}$. Sin embargo, extraoficialmente se estima que la producción total de pisco alcanzó 2,56 millones de litros en el 2005 y que dentro de diez años llegaría a 14 millones de litros (Olaechea, 2005). Los volúmenes de

2. Consultor internacional en márketing y representante de algunas firmas de pisco peruano en Estados Unidos, es el presidente de la cadena de venta al menudeo BevMax en dicho país, cadena que posee ochenta tiendas de licores en diferentes estados (teleconferencia realizada el 3 de marzo de 2006).

3. INEI (con cifras del Ministerio de la Producción y el Viceministerio de Industria). producción de pisco por bodega son, por lo general, pequeños y fluctúan entre 500 y 150 mil litros -o algo más-. Los volúmenes de producción en la mayoría de las bodegas pequeñas todavía no alcanzan economías de escala.

Aun cuando la calidad y el potencial internacional del pisco han sido reconocidos, la oferta exportable y su eficacia productiva en el Perú presentan diversos problemas. Estos han sido identificados por el Centro de Innovación Tecnológica Vitivinícola (Citevid), para el Programa de las Naciones Unidas para el Desarrollo (PNUD), y son los siguientes: a) escaso recurso hídrico en los valles de la costa del Perú, b) falta de fuentes de financiamiento a largo plazo para la agricultura, c) bajo nivel de educación, falta de capacitación técnica y ausencia de transferencia tecnológica y d) fragmentación de la propiedad, como consecuencia de la reforma agraria llevada a cabo en los años sesenta, hecho que originó la formación de minifundios con menos de una hectárea. Sin indicios del surgimiento de una asociación, todos estos problemas se traducen en mayores costos de producción.

\subsection{Exportaciones peruanas de pisco}

A pesar de la falta de competitividad y articulación de los actores participantes en el sector vitivinícola, la internacionalización del pisco peruano presenta un escenario favorable, dada la tendencia alcista que han registrado sus exportaciones, las cuales pasaron de 140 mil dólares a 458 mil dólares en los últimos seis años. En el año 2006 , solo el $13 \%$ de la producción de pisco se destinaba al mercado de exportación; el mercado interno constituía su principal destino. 
En el año 2005 se exportaron 82,7 mil litros de pisco; es decir, más de 9 mil cajas de 12 unidades de 750 mililitros cada una, volumen superior en 44,6\% al del año anterior. Por su parte, en el mismo periodo, Chile exportó 392 mil litros a un valor FOB de 863 mil dólares (ProChile y bUSiness Chile $^{4}$ ).

En cuanto al precio promedio, también se observa un incremento: el litro de pisco pasó de 2,2 dólares en el 2001 a 5,5 dólares en el 2005. Al analizar el comportamiento de las exportaciones, se asume que la recuperación del precio fue resultado de una mejora en la oferta exportable como consecuencia tanto del ingreso de productos de mayor calidad como del reconocimiento a la calidad del producto en los mercados externos.

Con relación a los destinos, en el 2005 el principal mercado receptor del pisco fue Estados Unidos, con una participación de $56,94 \%$ del total (equivalente a $257 \mathrm{mil}$ dólares), le siguieron Reino Unido, con $12,28 \%$, España, con $9,57 \%$, y Chile, con $3,15 \% 5$.

Para tener una idea de la participación relativa de la partida del pisco en el total de las exportaciones, se puede indicar que el valor FOB del total de las exportaciones peruanas en el 2005 ascendió a 17 mil

4. Programa de Fomento a las Exportaciones Chilenas (ProChile) <http://www.prochile.cl $>$ y Business Chile. Revista de AmCham Chile. $<$ http://www.businesschile.cl/portada.php? $\mathrm{w}=\mathrm{ol}$ $\mathrm{d} \& \mathrm{id}=136 \&$ lan $=\mathrm{es}>$.

5. Se encontró una variación entre los registros de la Superintendencia Nacional de Administración Tributaria (Sunat) y la Comisión para la Promoción de Exportaciones (Prómpex) con respecto al total de las exportaciones registradas en el 2005 y los países de destino. millones de dólares, las exportaciones no tradicionales alcanzaron 4280 millones de dólares, mientras que el valor correspondiente a la partida del pisco representó sólo el $1,06 \%$ de las exportaciones no tradicionales, es decir, 458 mil dólares ${ }^{6}$.

\subsection{Empresas exportadoras de pisco}

La agrupación en clusters de las empresas exportadoras a Estados Unidos ha sido realizada sobre la base de la clasificación de las empresas productoras de pisco del Ministerio de la Producción 7 . A continuación se presenta cada tipo de empresas y sus principales características.

\section{Empresas industriales}

Esta categoría la forman los siguientes productores industriales: Viña Tacama, Viña Ocucaje y Santiago Queirolo. Estas empresas se encuentran integradas verticalmente hacia atrás, pues poseen cultivos de uvas. Su principal giro de negocio es el vino, mientras que la producción de pisco es una actividad secundaria. Sus mayores esfuerzos se destinan a promover el desarrollo del mercado interno y a asegurar su participación en este, porque están en proceso de posicionamiento. Por esta razón, participan de la oferta exportable en forma limitada y su comportamiento es pasivo en este aspecto, pues sus exportaciones se generan por la demanda de los importadores de nacionalidad peruana en Estados Unidos, quienes les solicitan ser atendidos. Sus productos se orientan al mercado étnico, es decir, la colonia peruana, por medio de licorerías.

6. Según Prómpex, el valor FOB de las exportaciones ascendió a 16,9 millones de dólares en el 2005. Es decir, 33\% más respecto del 2004, que registró 12,7 millones de dólares.

7. TechnoServe-CITEvid, 2002. 
Las empresas industriales empezaron a participar en la oferta exportable a partir del 2002 y muestran un crecimiento continuo, aun cuando sus volúmenes comercializados son pequeños. Su participación en el volumen de las exportaciones totales a Estados Unidos en el 2005 fue de 18,28\% (Tacama, 1,84\%; Santiago Queirolo, 3,7\% y Ocucaje, 12,73\%).

\section{Empresas artesanales líderes}

En este grupo están consideradas las empresas pisqueras Agroindustrias Santo Tomás (pisco Montesierpe), César Chiarella, Puro Perú y Omnibeverages (pisco Gran Cruz), que producen principalmente para el mercado externo, con una percepción de negocio de largo plazo. Estas empresas son de tamaño mediano o pequeño, razón por la cual su disponibilidad de capital es menor, tienen escaso acceso al crédito y presentan problemas inherentes al manejo de empresas familiares o unipersonales, entre otras características representativas de su tamaño.

Si bien la presencia de estas empresas en la oferta exportable data del 2001, su participación en el total de exportaciones a Estados Unidos ascendió a $27,12 \%$ en el 2005 .

Estas empresas también tienen como mercado objetivo las colonias peruanas localizadas en diferentes estados, a quienes llegan por medio de la comercialización de sus productos en tiendas minoristas de licores. Al igual que el grupo anterior, trabaja con importadores regionales vinculados al mercado étnico.

\section{Qeros Perú}

Por último está Qeros Perú, empresa que ha sido clasificada en forma independiente porque sus exportaciones presentaron un comportamiento singular. El $95 \%$ de las ventas de esta empresa tiene como destino Estados Unidos. Así, en el 2005 sus exportaciones a dicho país representaron el $56 \%$ del total exportado por el Perú a ese destino, lo que la convirtió en la mayor empresa exportadora de pisco peruano a Estados Unidos. En su segundo año de exportaciones mostró un crecimiento de $235 \%$ en términos de volumen, logro que se atribuye al apoyo y asesoría del presidente de la cadena BevMax, Diego Loret de Mola, empresa minorista conocedora del mercado de licores y del consumidor estadounidense.

A diferencia de los otros tipos de empresas, Qeros se dirige al consumidor estadounidense, no al mercado étnico. Los bares, los restaurantes y los hoteles son los principales puntos de venta de sus productos, a los que presentan básicamente como ingredientes para la preparación de cocteles, en lugar de como licores puros. Las ciudades donde se distribuyen son conocidas como cosmopolitas. Es el caso de Miami, Nueva York, Connecticut y Las Vegas.

La empresa por medio de la cual Qeros ingresa a Estados Unidos es Shaw-Ross International Importers, considerada uno de los principales importadores de licores. Luego, los productos son distribuidos por los mayoristas de gran tamaño como Charmer, en Nueva York, Nueva Jersey y Connecticut; y Southern Wine \& Spirits, en Florida. 


\section{Distribución del pisco en Estados Unidos}

Como se ha visto en el punto anterior, los exportadores de pisco a Estados Unidos obtienen resultados positivos, pues muestran crecimiento tanto en los volúmenes de venta como en el valor exportado, ya sea atendiendo mercados étnicos o el estadounidense nativo. Sin embargo, para lograr mejores resultados en la comercialización del pisco peruano en dicho país, es necesario conocer al detalle los canales de distribución a los cuales está sujeto el producto como bebida alcohólica para poder llegar hasta el consumidor final.

\subsection{Marco legal para la comercialización de licores en Estados Unidos}

Toda persona o empresa que desee importar y/o comercializar bebidas alcohólicas en Estados Unidos debe obtener los permisos correspondientes ante tres organismos: la Food \& Drug Administration (FDA), que regula las normas sanitarias y medidas de seguridad alimenticia; el Bureau of Alcohol Tobacco \& Firearms (BATF), que otorga las licencias federales para la importación y regula el etiquetado del producto; y la State Liquor Control Board, organismo estatal que otorga la licencia del estado donde se distribuirán los productos (véase el gráfico 1).

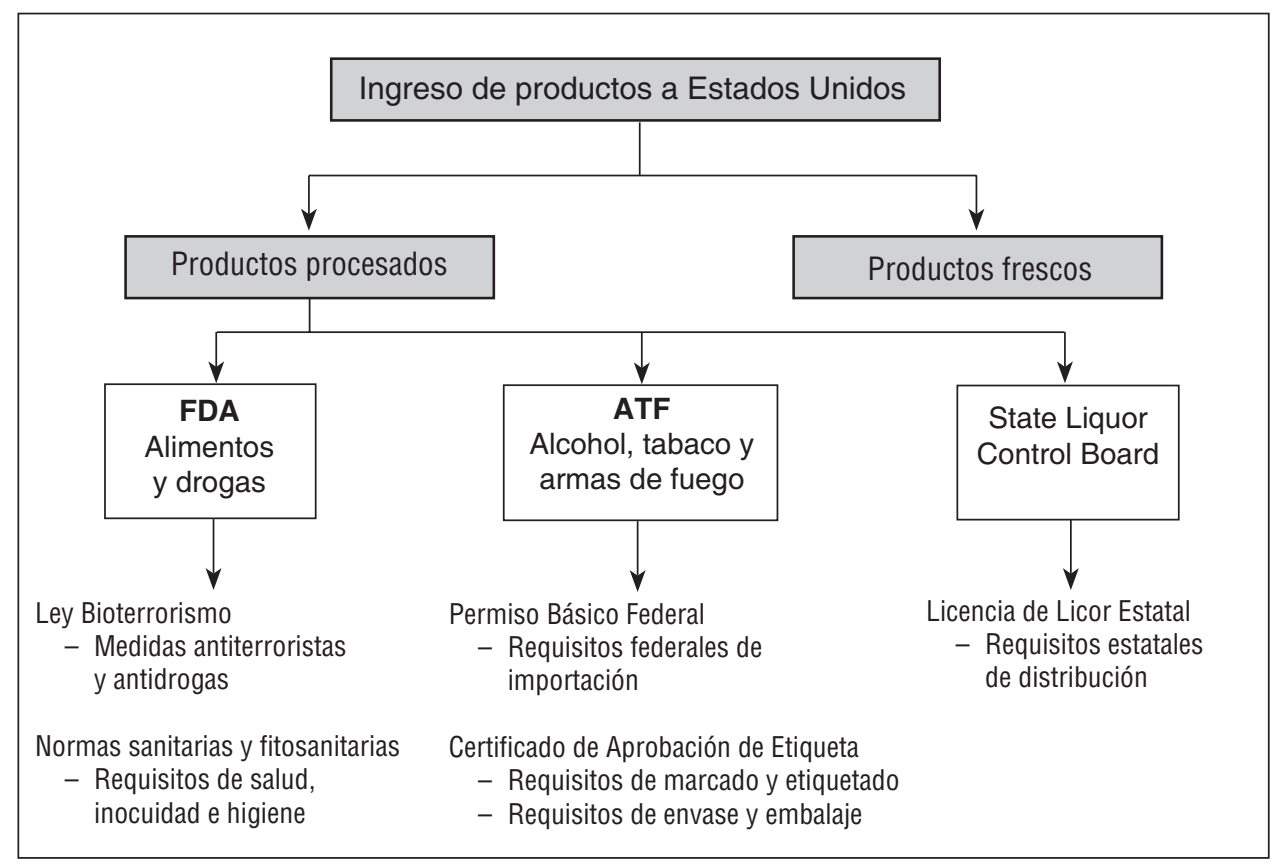

Fuente: García Díaz (2006).

Elaboración propia.

\section{Gráfico 1}

Descripción general de requisitos según organismo competente en Estados Unidos para la importación de bebidas alcohólicas 
Cabe señalar que si bien todos los estados controlan la venta de bebidas alcohólicas, dieciocho de ellos, denominados «estados de control», tienen el monopolio de la comercialización del alcohol a través de agencias de importación-distribución.

\subsection{Marco tributario}

En Texas, la bebida alcohólica debe pagar tres impuestos directos para ser comercializada: el federal, el estatal y el local; además de las cargas arancelarias impuestas al país de origen.

El impuesto federal (Federal Alcohol Excise Tax), conocido como Alcohol and Tobacco Tax, se aplica por el Gobierno Federal al alcohol y el tabaco. Sus tasas son específicas y varían en función del tipo de bebida y del grado alcohólico que esta contenga. En el caso de los licores espirituosos, este impuesto es de 13,50 dólares por proof gallon ${ }^{8}$ con $50 \%$ de contenido de alcohol. Para una botella de pisco peruano de 750 mililitros, con un contenido alcohólico de $40 \%$, el impuesto asciende a 2,14 dólares.
El importador paga este impuesto al momento de retirar los productos de la aduana (U.S. Customs and Border Protection) en el puerto de entrada del licor.

El impuesto estatal (State Alcohol Excise Tax) se aplica a todo licor importado que será comercializado por primera vez en el estado de Texas; Es decir, cuando un distribuidor mayorista poseedor de una licencia de importación comercializa el alcohol importado a minoristas o a un distribuidor local.

En el estado de Texas, la primera venta está sujeta al pago de un impuesto ascendente a 2,40 dólares por galón de bebida espirituosa, más $14 \%$ adicional en el caso de que la venta se realice en bares, restaurantes y hoteles (la llamada venta onpremise), es decir, 0,47 dólares por botella de 750 mililitros.

Cabe señalar que en otros estados puede haber impuestos adicionales, los cuales varían en monto o modalidad, esto es, pueden ser fijos o aplicarse como porcentaje del valor o el volumen del licor.

Precio por botella $=\frac{\left(\frac{U S \$ 13,50 \text { por galón }}{3,8 \text { litros }} \times 0,75 \text { litro por botella }\right) \times 40 \% \text { grado alcohólico }}{50 \% \text { grado alcohólico }}=$ US $\$ 2,14$

$$
\text { State Excise Tax (por botella) }=\left(\frac{\text { US\$ 2,40 }}{3,8 \text { litros }}\right) \times 0,75 \mathrm{ml} \text { por botella }=\text { US\$ 0,47 }
$$

8. Un galón es igual a 3,8 litros y a 3800 mililitros. Proof es el método para medir el contenido de alcohol en las bebidas espirituosas. El cálculo del proof de una bebida consiste en multiplicar el contenido de volumen alcohólico por 2. Por ejemplo: un producto con un contenido de volumen alcohólico de $40 \%$ tiene un proof de 80 [40 $\mathrm{x}$ por $2=80]$.
El estado y la ciudad determinan de manera individual los impuestos sobre las ventas. Generalmente, los impuestos estatales fluctúan entre $4 \%$ y $8 \%$, incluso algunas ciudades incluyen un impuesto adicional de $2 \%$. 
El impuesto local varía en función del estado en donde se comercializa el licor. En Texas, el impuesto es igual a un porcentaje sobre el valor de las ventas $(6,25 \%)$ más un impuesto local, que en la ciudad de Austin es de $2 \%$, lo que suma un total de $8,25 \%$.

Respecto a las cargas arancelarias, el pisco es uno de los productos peruanos beneficiados por la Ley de Preferencias Arancelarias Andinas (ATPA, por sus siglas en inglés), prorrogada y ampliada posteriormente por la Ley de Preferencias Arancelarias Andinas y de Erradicación de Drogas (ATPDEA, por sus siglas en inglés). En el tratado de libre comercio (TLC), próximo a ser firmado entre el Perú y Estados Unidos, se considera extender dichos beneficios ${ }^{9}$.

\subsection{Canales de distribución}

El canal de distribución constituye el factor crítico en el ingreso del pisco al mercado de Estados Unidos, pues de su diseño dependen otros aspectos que afectan el precio y la imagen del producto, así como la información que la exportadora pueda obtener del consumidor. Por su importancia, a continuación se analizan los canales de distribución utilizados desde que el producto es dejado por el exportador en el puerto de origen hasta que llega al consumidor final en el mercado de destino; incluidos los intermediarios y las funciones que cada uno cumple.

El importador, el mayorista-distribuidor y el minorista son los principales actores en el canal de distribución. Cada uno debe disponer de la licencia necesaria: el importador, la licencia federal; el distribuidor, la

9. Luego de 19 meses de negociaciones, los miembros de los equipos del Perú y Estados Unidos dieron por cerradas las discusiones para un TLC (Washington, 7 de diciembre de 2005). licencia estatal; y el minorista, la licencia local del minorista.

\subsubsection{El «sistema de tres capas» (The Three Tier System)}

El sistema de distribución está regulado por el «sistema de tres capas», diseñado con el fin de aislar a los comercios minoristas de las prácticas indeseables que ocurrían en el sector antes de la prohibición del alcohol. Entre ellas se puede mencionar aquellas que limitaban el acceso del minorista a una innumerable selección de marcas o lo obligaban a favorecer marcas de un proveedor en especial.

El actual «sistema de tres capas» (véase el gráfico 2) está diseñado de la siguiente forma: la primera capa la forman los productores y/o importadores de bebidas alcohólicas del mundo, los cuales venden sus productos a la segunda capa. En esta se ubican los distribuidores de bebidas o mayoristas poseedores de la licencia del estado para distribución, los que solo le venden a la capa tres.

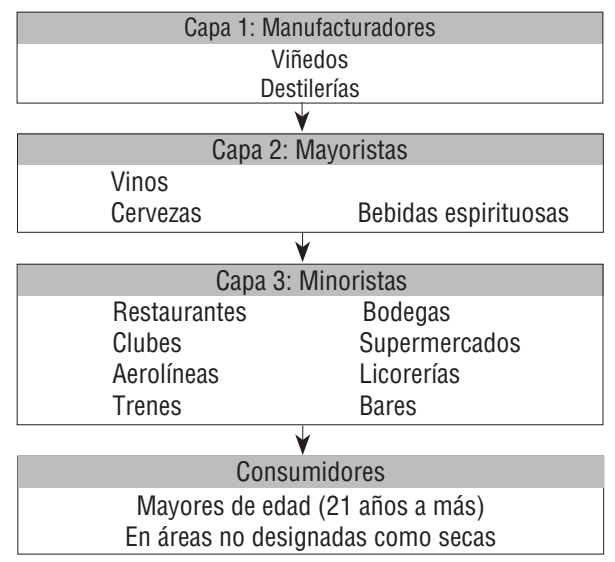

Fuente: Texas Safety Network. Elaboración propia.

Gráfico 2

El «sistema de tres capas» 
La tercera capa está integrada por los minoristas con licencias, quienes únicamente pueden vender bebidas alcohólicas a los clientes mayores de 21 años en las áreas donde está permitida la venta de alcohol.

Si bien este sistema se aplica en todo el país, cada estado aplica un procedimiento diferente para la concesión de licencias.

\subsubsection{Participantes en la cadena de distribución}

\section{Agente o broker}

Según la entrevista sostenida con Johnny Shuler (2006), el pisco peruano no se exporta de manera indirecta por medio de agentes o brokers.

\section{El importador}

El importador posee el conocimiento del mercado, los procedimientos burocráticos y las normativas federales y estatales aplicables. Dentro de la cadena, obtiene un margen promedio de $28 \%$ sobre el precio del exportador ${ }^{10}$.

El importador se encarga de solicitar al BATF la aprobación y el registro del producto que se importa (si es la primera vez) y es responsable del cumplimiento de la legislación relacionada con: envasado, marcaje, etiquetado, marcas comerciales, registro del producto, trámites aduaneros, entre otros.

La mayoría de importadores posee una red de distribución propia o designa a quienes serán sus distribuidores, los que a

10. ADEX. Seminario-taller «Las oportunidades comerciales del mercado de Canadá y USA para el pisco peruano». Lima, 2006. su vez disponen de una red de distribución minorista. El importador inicia y mantiene el circuito de comercialización de los productos en su área de competencia. Con el objetivo de focalizar los esfuerzos de márketing, especialmente cuando se trata de productos nuevos, el exportador y el importador pueden trabajar en conjunto para establecer una red de distribuidores en las áreas objetivo.

Una alternativa es el outsourcing con organizaciones que arriendan sus licencias de importación y se encargan de la liberación aduanera, la logística del transporte interno y el sistema de licencias en cada estado donde operan. Además, realizan los trámites de importación, almacenan stocks y envían los productos directamente a los distribuidores, de acuerdo con las instrucciones del exportador. En este caso, es responsabilidad de este último formar su propia red de distribuidores en Estados Unidos y negociar con ellos los contratos de venta. En estos casos, el «arrendador de licencia» trabaja por un honorario fijo o comisión.

\section{El mayorista-distribuidor}

Los distribuidores permiten la transferencia de las bebidas alcohólicas entre los proveedores y los minoristas en los estados. De acuerdo con la legislación vigente, en el caso de Texas el distribuidor debe ubicarse en Texas y los dueños deben ser residentes de ese estado (Texas Safety Network $^{11}$ ). Los distribuidores de Texas deben seguir las pautas y los procedimientos para la distribución de bebidas alcohólicas establecidos por la Texas Alcoholic

11. Texas Safety Network. <http://www.texassafety network.org/index.php>. 
Beverage Commission (TABC) $)^{12}$. En este estado, si bien el importador puede ser propietario del distribuidor, no le está permitido formar parte de la venta minorista. El margen del distribuidor es alrededor de $28 \%$.

Las funciones del distribuidor son: pagar el impuesto estatal sobre los productos vendidos, consolidar las órdenes para fabricantes e importadores, promover y mercadear las bebidas alcohólicas entre los minoristas, almacenar bebidas alcohólicas provenientes de todo el mundo, proveer a los minoristas de productos para atender la demanda del consumidor final, asegurar que el alcohol sea vendido sólo en las áreas permitidas y limitar la transferencia del alcohol de los distribuidores a los minoristas que obtuvieron correctamente sus licencias.

\section{El minorista}

Los minoristas representan la tercera capa del sistema y se clasifican en: tiendas de bebidas alcohólicas, que constituyen el principal canal de distribución, con el 50\% de participación de mercado; bodegas y supermercados, cuya participación de mercado es de $18 \%$, clubes privados, restaurantes y bares, que representan el $21 \%$ del mercado. Sin embargo, debido a que en este último caso el minorista transfiere al consumidor las bebidas a un precio elevado, su participación en términos de valor asciende a $57 \%$ de la cuota de mercado.

Las licencias para comercialización son tantas como los tipos de licores (cervezas, licores destilados, vinos, bebidas prepara-

12. Texas Alcoholic Beverage Commission es el organismo encargado de regular la distribución de bebidas alcohólicas en el estado de Texas. das). En ese sentido, cada minorista deberá comprarle al que posea la licencia correspondiente al giro de su negocio (tiendas minoristas, almacenes y supermercados, o restaurantes, bares y hoteles). En Texas, una de las restricciones del canal minorista es la prohibición de que las bodegas y supermercados distribuyan bebidas espirituosas. Debido a su grado alcohólico, estas bebidas solo se pueden expender en licorerías y bares-restaurantes ${ }^{13}$.

\section{Modelos de penetración del mercado}

\subsection{Análisis del canal de distribución}

Las organizaciones pueden diseñar diferentes cadenas de distribución y en número diverso. Sin embargo, es necesario escoger la que más se adapte a las necesidades del mercado, la actividad de la empresa y el tipo de producto.

Con el fin de determinar el canal más adecuado, se analizarán los canales de distribución para el pisco en el mercado de Estados Unidos, lo cual permitirá identificar los factores positivos y negativos de cada uno de ellos. En el gráfico 3 se resumen los posibles canales de distribución en ese país para la comercialización del pisco peruano.

\section{Canal 1: Importador-distribuidor- minorista}

En la cadena de distribución de este canal participan tres actores: el productor/exportador, que coloca sus productos a un importador; el importador autorizado, que puede

13. El grado alcohólico máximo permitido para comercializar un producto en supermercados y bodegas debe ser inferior a $17 \%$. 


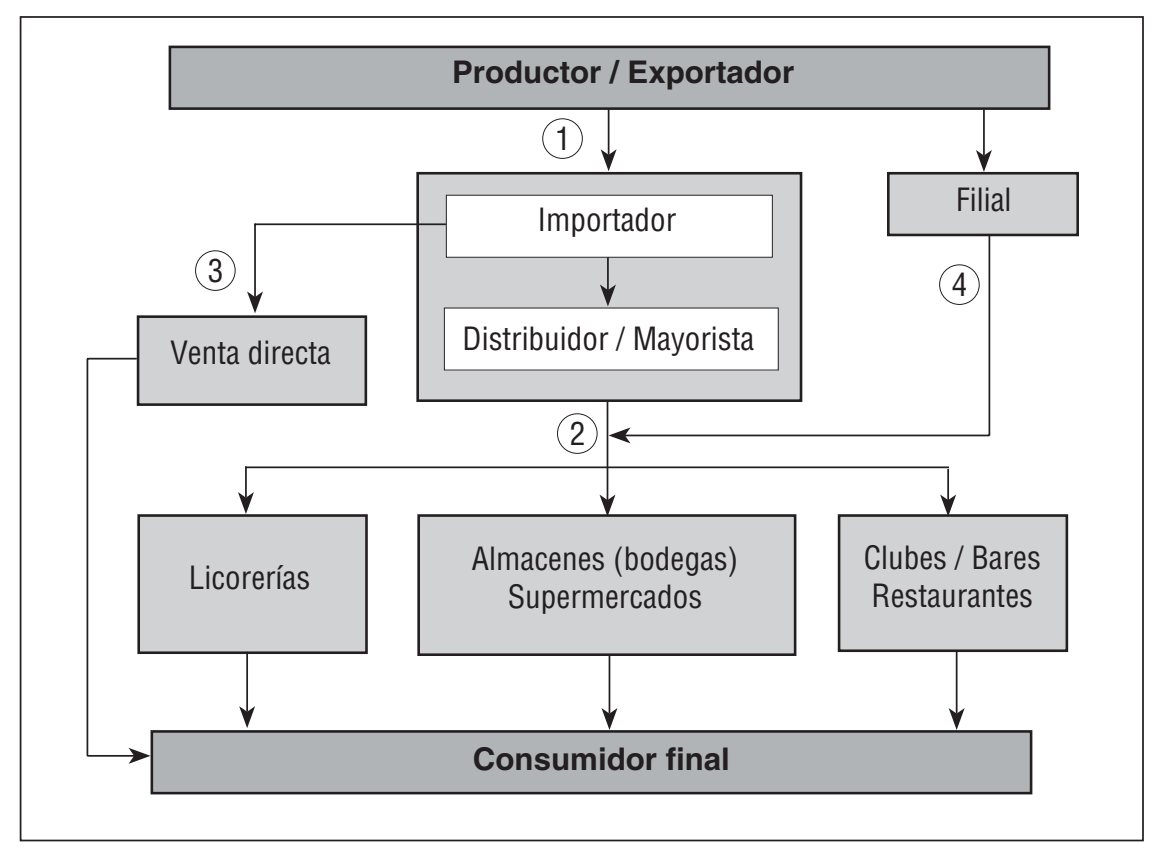

Elaboración propia.

Gráfico 3

Canales de distribución de licores en Estados Unidos

venderle sólo al distribuidor/mayorista, el que a su vez le vende a un minorista/detallista, adonde finalmente el consumidor acude a realizar la compra.

La ventaja de que el exportador use este canal es el apoyo del importador, que por su experiencia impulsa el producto, proporciona una red de distribuidores constituida y tiene mayor conocimiento del mercado.

La principal desventaja es el número de intermediarios que intervienen, pues el margen de cada uno de ellos encarece el producto hasta que llega al consumidor final. Además, cuanto mayor sea el tamaño del importador, mayor será su poder de negociación, en desmedro de los márgenes del exportador.

\section{Canal 2: Importador/distribuidor- minorista}

Al igual que en el caso anterior, el productor/exportador coloca su producto al importador; sin embargo, este actúa también como distribuidor y vende el producto a un minorista/detallista, adonde el consumidor final acude a realizar la compra. Este esquema es aplicable sólo en los estados que lo permiten, tal es el caso de Nueva Jersey, Florida y Texas.

La ventaja de este canal es el hecho de que el importador sea a su vez el distribuidor, pues de esa manera se logra desintermediar la cadena de distribución y reducir los márgenes que encarecen el producto final para el consumidor. 
La desventaja consiste en el alto poder de negociación del importador, el que en este modelo posee dos eslabones de la cadena de distribución y tiene mayor poder de decisión sobre el producto y la comercialización de este.

\section{Canal 3: Importador-distribución directa}

Según este modelo, el exportador coloca sus productos a un importador, el que establece una forma de distribución directa -por medio de Internet o por catálogo-con el consumidor. Si bien esta modalidad de distribución es aceptada en algunos estados, el de Texas no la permite.

La ventaja de esta modalidad se encuentra en el menor número de intermediarios dentro de la cadena, con la consecuente reducción de los costos y el aumento en los márgenes para el productor/exportador y el importador.

La desventaja radica en la legislación estatal, que prohíbe la distribución directa en algunos estados, lo cual representa un factor externo imposible de controlar. Además, la complejidad de garantizar que la cadena virtual, al igual que la cadena física, implemente los mismos procedimientos de seguridad en la comercialización encarece este canal. Entre los procedimientos de seguridad pueden mencionarse el verificar que los menores de edad no tengan acceso ilegal al alcohol ${ }^{14}$ y el que una dirección en particular no esté localizada en una comu-

14. En el 2004, la National Academy of Sciences publicó un informe que estimaba que el 10\% de los jóvenes encuestados admitió haber bebido alcohol siendo menor de edad y que la adquisición fue hecha por Internet o el sistema de entrega a domicilio. nidad en la cual no se permite la venta de bebidas alcohólicas.

\section{Canal 4: Filial importadora/ distribuidora-minorista}

El productor/exportador puede establecer una filial de la compañía en el país objetivo y ser este mismo el que se encargue de la importación de su producto. Dependiendo del tipo de licencia que obtenga y del estado en donde realice sus operaciones, el importador podrá desempeñar las funciones de distribuidor.

La ventaja principal es la reducción de intermediarios en la cadena de distribución, con el consecuente incremento de beneficios en términos de mayor margen para la empresa. Además, por la cercanía al mercado esta modalidad permite ejercer un elevado control de la comercialización, conocer las características del consumidor $\mathrm{y}$ tener mayor efectividad en las acciones de promoción.

La desventaja radica en que constituir la empresa en Estados Unidos puede ser una tarea difícil, ya que es necesario contar con un volumen de productos comercializables que permita obtener economías de escala y diluir costos fijos. Sin embargo, existe la opción de utilizar compañías de outsourcing para el almacenamiento de los productos, la constitución de la empresa y la gestión de cobranzas, de tal forma que los gastos fijos se reduzcan y se conviertan en variables.

\subsection{Determinación del precio (price scalation) en el canal de distribución 1}

La estructura de costos del pisco que se exporta a Estados Unidos varía en función 
de la cantidad de intermediarios que se utilice, el grado alcohólico del producto y el estado donde se distribuya.

Para analizar el precio del pisco peruano se ha tomado como ejemplo el caso particular del pisco Montesierpe, que se expende en la ciudad de Austin. Este ejercicio (véase el cuadro 1) permitirá construir el precio cada una, lo que da un total de 9 litros. El precio de exportación asciende a 34,68 dólares; y el precio FOB por botella, a 2,89 dólares. Las empresas aduaneras brindaron información sobre el flete y el seguro que cotizaron para un embarque en contenedor consolidado de 350 cajas de pisco (aproximadamente 4000 botellas) a Estados Unidos: 500 dólares o 0,12 dólares por botella.

\section{Cuadro 1. Cálculo del precio de venta al público del pisco peruano en Austin Presentación: caja de 12 botellas de 750 mililitros}

\begin{tabular}{|c|c|c|c|}
\hline & Canal de ventas: licorerías & Caja & Unidad \\
\hline & Precio neto del productor/exportador & 34,68 & 2,89 \\
\hline & + costo de flete & 1,43 & 0,12 \\
\hline \multirow[t]{2}{*}{$0,00 \%$} & + cargas arancelarias & - & - \\
\hline & = Costo del importador (valor CIF + arancel) & 36,11 & 3,01 \\
\hline $28,00 \%$ & + margen del importador/broker & 14,04 & 1,17 \\
\hline US\$ 2,14 & + impuesto federal (por botella) & 25,68 & 2,14 \\
\hline \multirow[t]{2}{*}{$8,25 \%$} & + impuesto al valor agregado, IVA (sobre costo importador + margen) & 4,14 & 0,34 \\
\hline & = Costo del distribuidor (= precio del importador) & 79,97 & 6,66 \\
\hline $28,00 \%$ & + margen del distribuidor (sobre su costo) & 31,10 & 2,59 \\
\hline US\$ 0,47 & + impuesto estatal (por botella) & 5,68 & 0,47 \\
\hline \multirow[t]{2}{*}{$8,25 \%$} & + IVA (sobre el margen) & 9,16 & 0,76 \\
\hline & $=$ Costo del minorista & 125,91 & 10,49 \\
\hline \multirow[t]{2}{*}{$25,00 \%$} & + margen del minorista (sobre su costo) & 41,97 & 3,50 \\
\hline & $\begin{array}{l}\text { = Precio del minorista (no incluye impuesto general } \\
\text { a las ventas, IGV) }\end{array}$ & 167,88 & 13,99 \\
\hline \multirow[t]{3}{*}{$8,25 \%$} & + IVA (sobre el margen) & 13,85 & 1,15 \\
\hline & = Precio del minorista (incluye IGV) & 181,73 & 15,14 \\
\hline & Precio final por botella de 750 mililitros & 15,14 & \\
\hline
\end{tabular}

Fuente: Trabajo de campo en Austin.

Elaboración propia.

final al consumidor (13,99 dólares) sobre la base del precio de exportación y adicionando los márgenes obtenidos por cada intermediario y los impuestos a los que está sujeto el producto.

El precio promedio del exportador se calcula sobre la base de la exportación de una caja de 12 botellas de 750 mililitros
El pisco Montesierpe, al igual que otras marcas encontradas en Austin, utiliza el canal de comercialización 1 , donde el margen del importador sobre el precio de venta es, en promedio, $28 \%{ }^{15}$, al igual que

15. Dato obtenido de entrevistas con empresarios del medio y de la presentación de Diego Loret de Mola en el IV Congreso Nacional del Pisco, realizado en Moquegua, en el 2005. 
el margen del distribuidor mayorista. El margen sobre el precio de venta promedio que obtiene el distribuidor minorista es de $25 \%$.

El precio final en el ejercicio comprueba los resultados obtenidos en el estudio de campo realizado en Austin, Texas, a partir de una muestra de licorerías que comercializan pisco Montesierpe, en las que se ofrece el producto al consumidor final a un precio de 13,99 dólares, antes del impuesto local.

Cabe mencionar que los bares y restaurantes obtienen un margen promedio de $200 \%$ porque el pisco se consume en la forma de cocteles (pisco sour, algarrobina, Perú libre y otros). El precio promedio de cada uno es de 4 dólares, y cada botella de 750 mililitros de pisco rinde 12 cocteles en promedio.

\section{Selección del modelo de penetración de mercado}

Para determinar el método de penetración en el mercado de Estados Unidos, se realizó la evaluación económico-financiera de cuatro tipos de proyectos de exportación: A) el modelo de la empresa Montesierpe, que utiliza el canal 1; B) el modelo de la empresa Qeros, el cual ha mostrado un crecimiento en ventas por encima del promedio; C) un modelo mixto, que es una combinación de los dos modelos anteriores, y D) un modelo bajo el esquema de promotor, el que realizaría las funciones de importación y distribución conjuntamente, para lo cual se utilizaría el canal 4.

Las variables críticas, que determinan las diferencias entre uno y otro modelo, se pueden observar en el cuadro 2. Son el nivel de crecimiento de las ventas, el precio FOB de exportación y el costo fijo destinado a la promoción.

Los gastos preoperativos son más altos para el modelo $\mathrm{D}$, por los costos en que se incurre para obtener las licencias correspondientes (importador y distribuidor) y los gastos de viajes al mercado de destino. En cambio, los modelos A y $\mathrm{C}$ tienen gastos preoperativos menores porque trabajan con importadores y no realizan mayor inversión. El nivel inicial de ventas se determinó en 4000 botellas para todos los modelos, con el fin de poder comparar los efectos de la modalidad de ingreso.

El incremento de las ventas es igual para todos los modelos: un crecimiento básico de $10 \%$ al año, con excepción de Qeros, que en dos años creció 193\%. Para realizar la evaluación, se utilizó un crecimiento anual de $30 \%$ para esta empresa.

Con relación al costo variable unitario, se supone que no existen ahorros por efectos de escala o aprendizaje, y que todos alcanzan los mismos costos de ventas. El modelo D asume costos altos por adquirir el producto terminado, pero no por producirlo.

El precio de venta por botella se basa en el registrado en el año 2005. Para los modelos D y C, el cálculo del precio consistió en un mark up del precio final (19,99 dólares) y de los intermediarios en la cadena.

Solo al modelo $\mathrm{C}$ se le aplicó una segunda etapa de crecimiento, como consecuencia del aprendizaje del mercado. Ello tuvo un impacto en el porcentaje de incremento de las ventas: de $10 \%$ a $30 \%$ a partir del sexto periodo, lo que implica un mayor gasto en promoción como costo fijo. 
Cuadro 2. Análisis de los modelos de canales de comercialización

\begin{tabular}{|c|c|c|c|c|}
\hline & $\begin{array}{c}\text { Modelo A: } \\
\text { Montesierpe }\end{array}$ & $\begin{array}{l}\text { Modelo B: } \\
\text { Qeros }\end{array}$ & $\begin{array}{c}\text { Modelo C: } \\
\text { Montesierpe } \\
\text { mejorado }\end{array}$ & $\begin{array}{l}\text { Modelo D: } \\
\text { Promotor }\end{array}$ \\
\hline \multicolumn{5}{|l|}{ Primera etapa } \\
\hline Gastos preoperativos & $(1000)$ & $(5000)$ & $(1000)$ & $(10000)$ \\
\hline Venta inicial & 4000 & 4000 & 4000 & 4000 \\
\hline$\%$ incremento de ventas & $10 \%$ & $30 \%$ & $10 \%$ & $10 \%$ \\
\hline Costo variable & 1,72 & 1,72 & 1,72 & 4,50 \\
\hline Precio FOB & 2,71 & 4,341 & 4,39 & 12,99 \\
\hline Costo fijo promoción & $(2000)$ & $(5000)$ & $(2000)$ & $(10000)$ \\
\hline Margen & $18,08 \%$ & $31,57 \%$ & $49,43 \%$ & $46,11 \%$ \\
\hline \multicolumn{5}{|l|}{ Segunda etapa } \\
\hline Inicio-periodo & - & - & 6 & - \\
\hline$\%$ incremento de ventas & $0 \%$ & $0 \%$ & $30 \%$ & $0 \%$ \\
\hline Costo fijo & - & - & $(5000)$ & - \\
\hline \multicolumn{5}{|l|}{ Resultados } \\
\hline Inversión capital & 7880 & 11880 & 7880 & 28000 \\
\hline VANE (valor actual neto) & 5780 & 88476 & 58805 & 140964 \\
\hline
\end{tabular}

Fuente: Trabajo de campo en Austin.

Elaboración propia.

El análisis económico nos muestra que todos los modelos obtienen resultados positivos (VANE $>0$ ). Sin embargo, el modelo que obtiene mejores resultados es el del importador/distribuidor, denominado «promotor» en el gráfico 4, que obtiene un VANE de 140 mil dólares con una inversión de 28 mil dólares, en un horizonte de evaluación de 10 años.

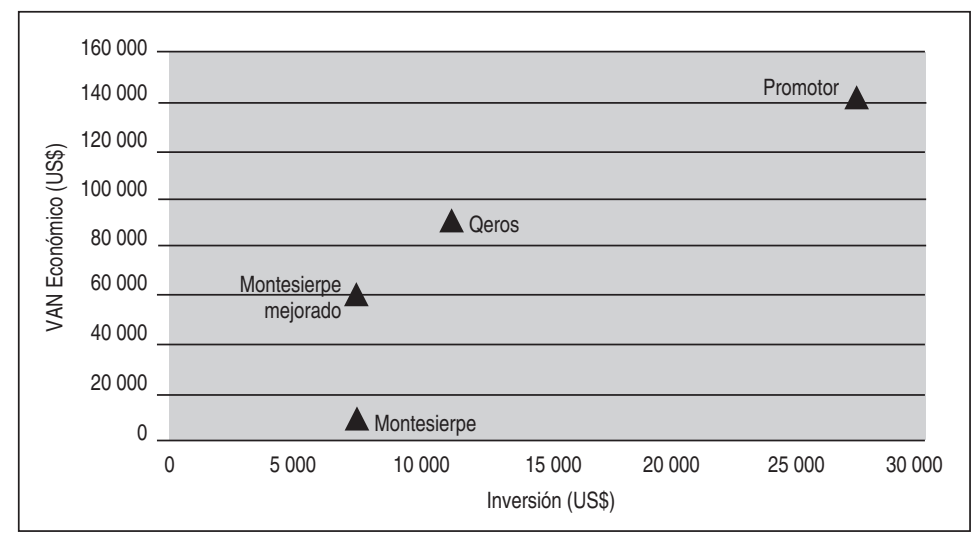

Fuente: Trabajo de campo, entrevistas en el Perú.

Elaboración propia.

\section{Gráfico 4}

Esquema representativo de inversión versus VANE

(en dólares) 
Por tanto, el método de penetración del producto que se implementará como canal de distribución es el modelo de importador/distribuidor, pues permite conseguir un resultado económico mayor. El modelo $\mathrm{D}$, denominado promotor, obtiene un resultado mejor pero, obviamente, implica no solo una mayor inversión de capital sino también asumir un riesgo mayor. Le sigue el modelo B, cuyo VANE es casi 50\% menor, debido a que la inversión también es aproximadamente tres veces menor. Cabe resaltar los resultados del modelo $\mathrm{C}$ en comparación con los del modelo A, como consecuencia del proceso de aprendizaje del mercado y el incremento en las ventas que experimenta en la segunda fase (véase el gráfico 4).

\section{Conclusiones}

El canal de distribución es un factor crítico para ingresar en el mercado estadounidense, ya que de su diseño dependen la determinación del precio del producto, la imagen, la información del consumidor, entre otros.

En la actualidad, el canal de distribución que utilizan los exportadores en Estados Unidos para el pisco peruano es el siguiente: importador-distribuidor-minorista. Este canal encarece el producto porque se paga, aproximadamente, un $30 \%$ de margen en cada uno de los eslabones de la cadena. La participación del exportador se limita a la entrega del producto en el puerto de origen, no va más allá en la cadena de distribución. Para obtener un mayor margen es necesario desintermediar la cadena, lo que aumentaría el monto de los márgenes por repartir. Después de realizar la evaluación financiera se determina que el método escogido de penetración en el mercado es el de inversión directa, mediante el uso del modelo de importador/distribuidor.

Cabe resaltar que la empresa Qeros ha aprovechado el aprendizaje del asesor Loret de Mola, quien ha transferido a la empresa sus conocimientos del mercado y del comportamiento del consumidor estadounidense. Por tanto, esta empresa obtiene ratios de crecimiento superiores a los de otros exportadores, así como un mayor retorno.

En cuanto al marco legal y tributario, las leyes que regulan la comercialización de bebidas alcohólicas en Estados Unidos están dadas por tres organismos: la Food \& Drug Administration (FDA), que regula las normas sanitarias y de bioterrorismo; el Alcohol Tabacco \& Firearms (ATF), que otorga las licencias federales para importación, empaquetado y etiquetado del producto; y la State Liquor Control Board, organismo estatal que otorga la licencia del estado donde se distribuirán los productos.

Un factor de éxito reside en conocer las regulaciones y los requerimientos legales y tributarios del mercado de licores en Estados Unidos, debido a la compleja estructura normativa federal, estatal y local. En ese sentido, antes de realizar esfuerzos de penetración en el mercado de Estados Unidos, se debe focalizar la estrategia de ingreso en un estado o un número limitado de estados y evaluar qué normativas son las más recomendables y/o favorables en cada uno de ellos. 


\section{Referencias bibliográficas}

ALCOHOL AND TABACCO TAX AND TRADE BUREAU. <http://www.ttb.gov/ espanol/sobre_ttb.htm $>(12-1-2006)$.

ALDEA, Cristina. 2004. El mercado de las bebidas espirituosas de EE. UU. Cámara de Comercio e Industria de Zaragoza. <http:// www.camarazaragoza.com/publicaciones. asp $>(31-1-2006)$.

\section{CENTRO DE INNOVACIÓN TECNOLÓGICA} VITIVINÍCOLA (CITEvid). 2004. La uva y el pisco: potencialidades productivas. Programa de las Naciones Unidas para el Desarrollo (PNUD).

DEUSTÚA, Ana María. (s. f.). Requisitos para la importación de pisco en los Estados Unidos. Oficina Comercial del Perú en Nueva York.

GARCÍA DÍAZ, J. A. 2006. Oportunidades de exportación a Estados Unidos. Caso: pisco peruano. Seminario-Taller «Las oportunidades comerciales del mercado de Canadá y USA para el Pisco Peruano». Conferencia dictada en ADEX, Lima, 19 de enero de 2006.
LORET DE MOLA, Diego. 2006. Teleconferencia con Diego Loret de Mola, director de BevMax en Estados Unidos, realizada el 3 de marzo.

MARKET RESEARCH CENTRE AND THE CANADIAN TRADE COMMISSIONER SERVICE. 2002. The Alcoholic Beverage Market in the United States.

OLAECHEA, P. 2005. La industria vitivinícola nacional. Conferencia dictada en el IV Congreso Nacional de Pisco, Moquegua.

PACULT, F. P. High Spirits, Low Prices. Wine Enthusiast Magazine. New York, Nov. 15, 2005. <www.winemag.com>.

PERÚ. INSTITUTO NACIONAL DE DEFENSA DE LA COMPETENCIA Y DE LA PROTECCIÓN DE LA PROPIEDAD INTELECTUAL (INDECOPI). 2006. Bebidas alcohólicas. Pisco. Requisitos. Comisión de Reglamentos Técnicos y Comerciales, NTP 211.001, 2 de noviembre de 2006. 